\title{
Overexpression of oxidored-nitro domain containing protein 1 inhibits human nasopharyngeal carcinoma and cervical cancer cell proliferation and induces apoptosis: Involvement of mitochondrial apoptotic pathways
}

\author{
JUE OUYANG ${ }^{1,3}$, MINGHUA WU ${ }^{1,3}$, CHEN HUANG $^{2}$, LI CAO $^{1}$ and GUIYUAN LI ${ }^{1,3}$ \\ ${ }^{1}$ Cancer Research Institute, Disease Genome Research Center, Central South University; Key Laboratory \\ of Carcinogenesis and Cancer Invasion, Ministry of Education; Key Laboratory of Carcinogenesis, \\ Ministry of Health, Changsha, Hunan; ${ }^{2}$ Medical Research Center, Peking University Third Hospital, \\ Beijing; ${ }^{3}$ Key Laboratory of Nonresolving Inflammation and Cancer, Changsha, Hunan, P.R. China
}

Received June 19, 2012; Accepted August 7, 2012

DOI: 10.3892/or.2012.2101

\begin{abstract}
Oxidored-nitro domain containing protein 1 (NOR1) is a novel member of the nitroreductase family that was first isolated as a tumor suppressor gene from human nasopharyngeal carcinoma (NPC). However, the role of NOR1 gene dysfunction in human cancers has not been addressed. We analyzed the expression of NOR1 in various human cancer and benign tissue specimens and found significant downregulation in nine types of cancer compared with corresponding non-tumor tissues. The recombinant expression vector pCDNA3.1-mychis-NOR1 was constructed and transfected into human NPC 6-10B nasopharyngeal cancer and HeLa cervical cancer (CCA) cells. We found that stable NOR1 overexpression resulted in suppression of 6-10B and HeLa cell proliferation and led to $\mathrm{S}$ phase cell cycle arrest. In addition, NOR1 upregulation enhanced apoptosis in pCDNA3.1-myc-his-NOR1 stably transfected cells, and it also altered the expression of proteins involved in the mitochondria-dependent apoptotic pathway. Furthermore, we also found that the NOR1 protein is a cytoplasmic protein that is partially localized in the mitochondria and endoplasmic reticulum. Therefore, NOR1 is an important tumor suppressor gene associated with NPC and CCA and may play antitumor roles by inhibiting proliferation, preventing colony formation, and promoting the apoptosis of tumor cells via the mitochondrial-dependent apoptotic pathway. However, the precise mechanism behind the NOR1 antitumor effects needs to be investigated further.
\end{abstract}

Correspondence to: Dr Guiyuan Li, Cancer Research Institute, Central South University, Xiangya Road, Changsha, Hunan 410078, P.R. China

E-mail: ligy@xysm.net

Key words: oxidored-nitro domain containing protein 1,proliferation, apoptosis, mitochondria, nasopharyngeal carcinoma, cervical cancer

\section{Introduction}

The oxidored-nitro domain-containing protein 1 (NOR1) gene (also known as OSCP1) is a novel tumor suppressor gene that was first isolated from a nasopharyngeal carcinoma (NPC) (1). Human NOR1 expression is decreased in the HNE1 NPC cell line (1) and tissues when compared with normal nasopharyngeal epithelial cells (2). Furthermore, mutations in the NOR1 coding region were found in NPC biopsies (1), and a hypermethylated promoter region of NOR1 was found in NPC biopsies (3), leukemia cell lines, and acute myeloid leukemia patients (4). Our previous study has shown that the functional NOR1 promoter is regulated by heat shock factor 1 (HSF1) and nuclear respiratory factor 1 (NRF1) and the promoter is located within a CpG island (3). Hypermethylation of this $\mathrm{CpG}$ island was found in NPC tissue samples and cancer cell lines, whereas no aberrant promoter methylation was detected in non-cancerous nasopharyngeal tissue samples or normal nasopharyngeal epithelial cells. Promoter hypermethylation may occur during transcriptional inactivation of the NOR1 gene in cancer cells and NOR1 may be a critical tumor suppressor involved in the development of various cancers.

The human NOR1 gene is located on 1p34.3 and shares $40 \%$ homology with nitroreductases from Escherichia coli. This homology implies that NOR1 may be a novel member of nitroreductases, a group of FMN- or FAD- and NAD(P) $\mathrm{H}$-dependent enzymes, which catalyze the reduction of nitro groups in a wide range of substrates to produce the corresponding hydroxylamine (5). Since much evidence has shown that exposure to nitroso compounds such as nitrosamines is a risk factor for NPC (6-9), NOR1 may play an important role in chemical carcinogen formation and NPC carcinogenesis due to its nitrosation function. With a highly specific NOR1 antibody, Xiang et al demonstrated that NOR1 is predominantly expressed in the nasopharynx and trachea and is weakly expressed in the central nervous system (2). These data explain the selectivity of the potential NOR1 physiological functions and provide an indispensable marker for the NPC 
carcinogenesis process, and the identification or validation of tissue-specific drug targets may help in the further study of the selectivity of the organ targets that respond to chemical carcinogens such as nitroso compounds. However, to date, the tumor suppression functions of NOR1 have not been completely elucidated.

In this study, we performed NOR1 expression analysis in various human cancer and benign tissue specimens and analysis of the antitumor effects of NOR1 using in vitro cell functional studies. We demonstrate that NOR1 expression was significantly decreased in nine types of cancer tissues: ovary, lung, kidney, vulva, prostate, uterus, cervix, thyroid gland, and testis. The exogenous NOR1 overexpression results in inhibited proliferation and colony-forming ability of NPC 6-10B and CCA HeLa cells and leads to $\mathrm{S}$ phase cell cycle arrest.

In addition, NOR1 upregulation enhanced apoptosis under the stable transfection of pCDNA3.1-myc-his-NOR1. NOR1 overexpression activated caspase 9 , released cytochrome $\mathrm{c}$ from mitochondria to cytoplasm, increased Bax and p53 expression, and decreased Bcl-2 expression, which is involved in the mitochondria-dependent apoptotic pathway. Furthermore, we also found that NOR1 is a cytoplasmic protein that is partially localized to the mitochondria and endoplasmic reticulum. Therefore, NOR1 is an important tumor suppressor gene that is related to NPC and CCA and may play antitumor roles by inhibiting proliferation, preventing colony formation, and promoting the apoptosis of tumor cells via the mitochondria-dependent apoptotic pathway.

\section{Materials and methods}

Reagents. XhoI, BamHI, and primers and probes were purchased from Takara (Dalian, China). The Cancer Profiling Array II was purchased from BD Biosciences Clontech (Palo Alto, CA, USA). The DIG Olignucleotide 3'-tailing labeling kit and anti-DIG-POD were purchased from Roche (Germany). The RevertAid $^{\mathrm{TM}}$ First Strand cDNA synthesis kit and PCR Master mix (2X) were purchased from Fermentas (Glen Burnie, MD). The RPMI-1640 medium, fetal bovine serum (FBS), pcDNA3.1myc-his plasmid, Lipofectamine 2000, TRIzol, MitoTracker Red CMXRos, ER-Tracker Red, and Opti-Mem I were purchased from Invitrogen (Carlsbad, CA, USA). Gentamicin-G418, the endoplasmic reticulum isolation kit, and all other chemicals were obtained from Sigma (St. Louis, MO, USA). The BCA protein assay and Mitochondria isolation kit were purchased from Pierce (Rockford, IL).

The ECL system was purchased from Amersham Pharmacia Biotech (Piscataway, NJ). The antibodies used, obtained from Abcam (Cambridge, UK), were as follows: anti-His (clone HIS. H8, dilution 1:1500); anti- $\beta$-actin (clone mAbcam 8226, dilution 1:2000); anti-Bax (clone 6A7, dilution 1:1000); anti-Bcl-2 (clone 100/D5, dilution 1:1000); anti-p53 (clone pAb 240, dilu-

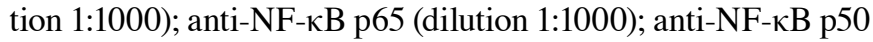
(dilution 1:1000); anti-cytochrome c (clone EP1326Y, dilution 1:1000); anti-caspase 9 (dilution 1:1000); HRP-conjugated goat anti-rabbit IgG (dilution 1:3000); and HRP-conjugated goat anti-mouse IgG (dilution 1:3000).

Cell culture and transfection. The HNE1 Human NPC and the HeLa CCA cell lines were maintained in RPMI-1640 supple- mented with $10 \%$ FBS in a humidified incubator with $5 \% \mathrm{CO}_{2}$ at $37^{\circ} \mathrm{C}$.

For stable transfection, a mammalian expression system using the pCDNA3.1-myc-his-NOR1 plasmid was created by inserting the full-length human NOR1 cDNA, which was obtained from IMAGE Consortium, between the XhoI and BamHI sites of the pcDNA3.1-myc-his plasmid. The transfection experiment was performed with a pcDNA3.1-myc-his plasmid containing the full-length NOR1 cDNA insert or with a pcDNA3.1-myc-his plasmid containing no insert using Lipofectamine 2000 according to the protocol of the manufacturer. Gentamicin-G418 $(500 \mu \mathrm{g} / \mathrm{ml})$ was added for transfectant selection. The stable transfectants were isolated after 3 weeks using a pooled-cloning technique. Cells that grew to $80 \%$ confluence were harvested for experiments.

The transient transfection experiment was performed with a pGFP-N1-NOR1 vector (a gift from Dr Xinming Nie) containing the full-length human NOR1 cDNA and a control vector using Lipofectamine 2000 according to the manufacturer's protocol. After transfection for $48 \mathrm{~h}$, the cells were used in subsequent experiments.

Cancer profiling array. The differential expression of NOR1 mRNA in normal and cancer tissues from 19 different tissues was determined using the Cancer Profiling Array II using cDNA from paired cancer and normal human tissues. This array includes 154 pairs of cDNA blots prepared from the full thickness of cancer specimens from 19 different tissues, each paired with cDNA from normal tissue from the same patient. Pre-hybridization, hybridization, and post-hybridization washings were performed according to the protocol of manufacturer. A ${ }^{32} \mathrm{P}$-labeled NOR1-specific cDNA probe was used for hybridization. The array was then exposed to a storage phosphor screen (Molecular Dynamics, Amersham Biosciences) for $36 \mathrm{~h}$ at room temperature. A Storm 840 phosphorimager system (Molecular Dynamics, Amersham Biosciences) and ImageQuant software version 4.1 (Molecular Dynamics, Amersham Biosciences) were used to quantify the hybridization signals.

Tissue microarrays and in situ hybridization. The tissue microarrays (TMAs), which were prepared from NPC and normal tissue specimens collected at the Second Xiangya Hospital (Changsha, Hunan, China), were constructed in our laboratory. The antisense sequence of three in situ hybridization (ISH) nucleotide probes from different regions of the NOR 1 cDNA used were: 5'-ccttcttggagtagagctcttgaggcttga-3' (325-354 bp); 5'-gaactccctgcagagagaccaccatata-3' (594-623 bp); 5'-catagga taactcttctggcctggttagcg-3' (1126-1155 bp).

The probes were labeled with 11-DIG-dUTP at their $3^{\prime}$ tails. The ISH detection method was performed according to protocol of the manufacturer. Briefly, the sections were deparaffinized, rehydrated, digested with $2 \mu \mathrm{g} / \mathrm{ml}$ proteinase $\mathrm{K}$ at $37^{\circ} \mathrm{C}$ for $15 \mathrm{~min}$, and dehydrated. The slides were incubated with prehybridization solution at $37^{\circ} \mathrm{C}$ for two hours and hybridized with the NOR 1 probe at $37^{\circ} \mathrm{C}$ overnight. Hybridization was detected by incubation with anti-digoxigenin horseradish peroxidase (anti-DIG-POD) fab fragments. The color reaction was performed with 3.3'-diaminobenzidine tetrahydrochloride (DAB), and the slides were counterstained with hematoxylin. 
A poly $\mathrm{d}(\mathrm{T})$ oligo was used as a control for total RNA preservation. The GAPDH housekeeping gene and prehybridization solution containing no probe were used as positive and negative control, respectively. The NOR1 positive hybridization signals were microscopically scored at $\mathrm{x} 400$ magnification. The scoring was recorded in accordance with the following staining proportion and intensity: 0 (negative), 1 ( $<10 \%$ nuclei being EBER-1 positive), 2 (10-50\% positive), or 3 ( $>50 \%$ positive). The final scores were regarded as negative (0 score) and positive (1-3 score).

RNA extraction and reverse transcriptase-polymerase chain reaction $(R T-P C R)$. Total RNA was isolated using TRIzol according to the manufacturer's protocol. Total RNA $(5 \mu \mathrm{g})$ was reverse transcribed with reverse transcriptase (RevertAid ${ }^{\mathrm{TM}}$ First Strand cDNA synthesis kit) and used for PCR using PCR Master Mix (2X). The primers for NOR1, GAPDH, and $\beta$-actin were: NOR1 5'-ACCTGCACATCCGAGTATCC-3' (forward) and 5'-CTGGCCAAGAAATTCAGCTC-3' (reverse); GAPDH 5'-ATGTTCGTCATGGGTGTGAA-3' (forward) and 5'-TGC TGTAGCCAAATTCGTTG-3' (reverse); $\beta$-actin 5'-CCTCG CCTTTGCTGATCC-3' (forward) and 5'-GGATCTTCATGA GGTAGTCAGTC-3' (reverse).

Protein isolation and western blotting. Cells at $80 \%$ confluence were harvested for western blot analysis. The harvested cells were lysed, and their protein concentrations were determined using a BCA protein assay. The cell lysates $(50 \mu \mathrm{g}$ of protein each lane) were separated by $10 \%$ sodium dodecyl sulfate-polyacrylamide gel electrophoresis (SDS-PAGE) and transferred to nitrocellulose membranes. The membranes were blocked with $5 \%(\mathrm{v} / \mathrm{v})$ skim milk and probed with primary antibody at $4{ }^{\circ} \mathrm{C}$ overnight. After washing, the membranes were incubated with HRP-conjugated secondary antibody at room temperature for $1 \mathrm{~h}$. The primary antibodies used were specific for human His, HIF-1 $\alpha, \beta$-actin, Bax, Bcl-2, p53, NF- $\kappa$ B p65, NF- $\kappa$ B p50, cytochrome $\mathrm{c}$, and caspase 9 . The bound antibodies were visualized using the ECL system.

Cell growth assay. Cell proliferation was characterized by a 3-[4,5-dimethylthiazol-2-yl]-2,5-diphenyltetrazolium bromide (MTT) assay. Cells ( $10^{4}$ cells/well) were incubated with RPMI1640 containing $10 \%$ FBS in 96-well plates for seven days. MTT ( $20 \mu \mathrm{l}$ of $5 \mathrm{mg} / \mathrm{ml}$ ) was added daily to each well and incubated at $37^{\circ} \mathrm{C}$ for $4 \mathrm{~h}$, after which the MTT solution in the medium was aspirated off. To achieve solubilization of the formazan crystals formed in viable cells, $150 \mu \mathrm{l}$ of DMSO was added to each well. Cell proliferation was determined by measuring the converted formazan at $570 \mathrm{~nm}$ using an ELX-800 ELISA plate reader (Bio-Tek Instruments, Winooski, VT).

Soft agar colony forming assay. For soft agar assays, a bottom layer of $1 \mathrm{ml}$ of the corresponding culture media containing $0.6 \%$ agar and $10 \%$ fetal calf serum was prepared, placed in $35-\mathrm{mm}$ culture dishes, and allowed to solidify. Cells $\left(2 \times 10^{4}\right)$ were suspending in $50 \mu \mathrm{l}$ of RPMI-1640 containing $10 \%$ FBS and G418 $(500 \mu \mathrm{g} / \mathrm{ml})$. Culture medium $(1 \mathrm{ml})$ containing $0.33 \%$ agarose was added to the cell suspension before seeding the dishes. Triplicates were performed for each cell type. Cells were incubated at $37^{\circ} \mathrm{C}$ in a $5 \% \mathrm{CO}_{2}$ atmosphere. The dishes were examined twice per week, and the colonies were manually counted after 2 weeks.

Cell cycle and apoptosis assay. Cell cultures were plated in 10 -cm dishes at $\sim 40 \%$ confluence and allowed to grow exponentially. The adherent cells were collected by trypsinization, pooled with the non-adherent cells, and washed with phosphate-buffered saline (PBS). The cells were then fixed in $70 \%$ cold ethanol overnight at $4^{\circ} \mathrm{C}$. Before analysis, the cells were adjusted to a final density of $1 \times 10^{6}$ cells $/ \mathrm{ml}$ in PBS containing RNase $(1 \mu \mathrm{g} / \mathrm{ml})$ and stained with $10 \mu \mathrm{g} / \mathrm{ml}$ propidium iodide for $30 \mathrm{~min}$ at room temperature. The multiparameter analysis of 5000 cells was performed on a FACScan flow cytometer using CellQuest software (BD Biosciences, San Jose, CA, USA).

Acridine orange $(A O) /$ ethidium bromide $(E B)$ staining. An $\mathrm{AO} /$ EB cocktail $(80 \mu \mathrm{l})$ containing $1 \mathrm{ml}$ of RPMI-1640 was added to the culture plate. Fields of stained cells were selected and focused on using fluorescence microscopy (Nikon Eclipse E800; Nikon, Tokyo, Japan). Viable cells, which were stained with only $\mathrm{AO}$, were bright green with intact structure; early apoptotic cells, which were stained with $\mathrm{AO}$ and $\mathrm{EB}$, were bright green in the nucleus with red-orange chromatin. Late apoptotic cells, which were stained with both $\mathrm{AO}$ and $\mathrm{EB}$, were red-orange with chromatin condensation (10).

RNA interference (RNAi) treatment. The HIF-1 $\alpha$ siRNA oligonucleotides used were: 5'-ATGGAGGGCGCCGGC-3' (sense) and 5'-GCCGGCGCCCTCCAT-3' (antisense). On the day of RNAi transfection, the media were removed from $50 \%$ confluent cells cultured in a 12 -well plate, and $800 \mu \mathrm{l}$ of Opti-Mem I was added. Then, HIF-1 $\alpha$ siRNA was diluted in Opti-Mem I to a final volume of $200 \mu \mathrm{l}$ containing $2 \mu \mathrm{l}$ of Lipofectamine 2000 reagent (Invitrogen) and incubated at room temperature for $25 \mathrm{~min}$ before addition to each well. The final working concentration of HIF- $1 \alpha$ siRNA was $100 \mathrm{nM}$. Twenty-four hours later, the culture medium was replaced with RPMI-1640 without FBS. The cells were used for the following assays.

Mitochondria and endoplasmic reticulum (ER) isolation. Cells were grown to $80-90 \%$ confluence and collected by trypsinization into tubes $\left(4 \times 10^{7}\right.$ cells per sample). The mitochondria were isolated using the mitochondria isolation kit, and the ERs were separated using the Endoplasmic Reticulum Isolation kit according to the manufacturer's protocol.

Mitochondria and ER staining. Cells were grown to $70 \%$ confluence on a coverslip. Mitochondria were stained with $50 \mathrm{nM}$ MitoTracker Red CMXRos at $37^{\circ} \mathrm{C}$ for $20 \mathrm{~min}$, and the cells were washed twice with PBS and fixed with freshly prepared $4 \%$ formaldehyde at $37^{\circ} \mathrm{C}$ for another $20 \mathrm{~min}$. The ER was stained with $1 \mu \mathrm{M}$ ER-Tracker Red for $30 \mathrm{~min}$ at $37^{\circ} \mathrm{C}$, and the cells were washed twice with PBS without fixation. Cell images were captured with an LSM 510 Zeiss confocal microscope.

Northern blotting. Cells at $80 \%$ culture confluence were harvested, the total RNA was extracted, and $40 \mu \mathrm{g}$ per sample was dotted onto a nitrocellulose membrane. The membranes 


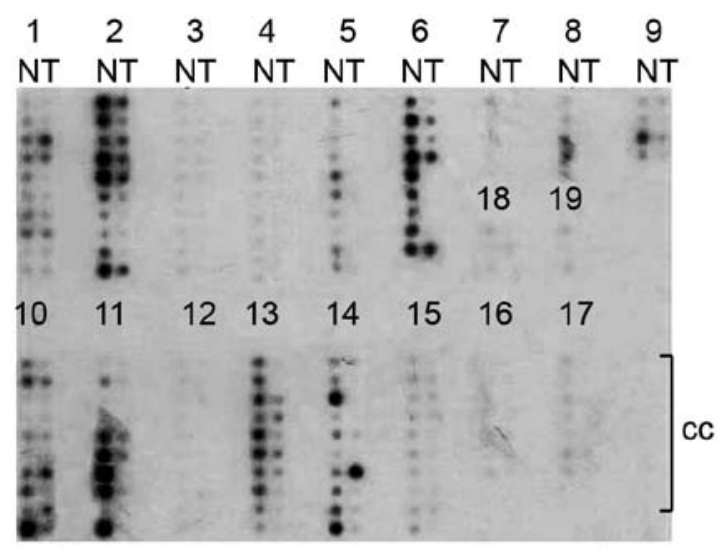

Figure 1. Expression of NOR1 was demonstrated by the cancer profiling array II. The Cancer Profiling Array II was hybridized with a NOR1radiolabeled probe. Hybridization signals were detected by phosphorimager. The numbers indicate the tissue types in lanes: 1) breast, 2) ovary, 3) colon, 4) stomach, 5) lung, 6) kidney, 7) bladder, 8) vulva, 9) prostate, 10) uterus, 11) cervix, 12) rectum, 13) thyroid gland, 14) testis, 15) skin, 16) small intestine, 17) pancreas, 18) trachea, and 19) liver. N, normal; T, tumor; cc, cancer cell line cDNA.

were then prehybridized with $100 \mu \mathrm{g} / \mathrm{ml}$ salmon sperm DNA in a solution containing $6 \mathrm{X}$ SSC, $0.5 \%$ SDS, $5 \mathrm{X}$ Denhardt's at $68^{\circ} \mathrm{C}$ for $3 \mathrm{~h}$. The membranes were hybridized with ${ }^{32} \mathrm{P}$-labeled PCR-amplified NOR1- or 28S-specific probes at $68^{\circ} \mathrm{C}$ for $24 \mathrm{~h}$. The membranes were then washed twice with $2 \mathrm{X} \mathrm{SSC} / 0.1 \%$ SDS at room temperature for $15 \mathrm{~min}$ followed by a $30 \mathrm{~min}$ wash with $0.1 \mathrm{X} \mathrm{SSC} / 0.1 \%$ SDS. Subsequently, the filters were exposed to X-ray film (Kodak BioMax) for $48 \mathrm{~h}$ at $-80^{\circ} \mathrm{C}$. The relative abundance of the individual mRNAs in each clone was normalized to the density of the $28 \mathrm{~S}$ rRNA dot.

Immune electron microscopy. Immune electron microscopy was carried out as described previously (10). The cells were fixed with $2 \%$ paraformaldehyde, subsequently dehydrated, embedded, and polymerized under UV light at room temperature for 48-72 h. Ultrathin sections were cut and collected on pioloform-coated nickel grids to continue the immunolabeling experiments. The primary antibody was anti-His antibody and the diameter of colloidal gold immune complexes (1:10, Boster Bio-Engineering Ltd. Wuhan, P.R. China) was $10 \mathrm{~nm}$. The dried sections were then stained with sodium acetate and observed under a transmission electron microscope.

Statistical analysis. The data are presented as the means \pm SE. Differences of the variables between the groups were analyzed by the Student's t-test or a one-way ANOVA test. Differences were considered significant when $\mathrm{P}<0.05$.

\section{Results}

Underexpression of NOR1 mRNA in various cancer types. To examine NOR1 expression in various types of cancer, the NOR1 probe was labeled and hybridized to a commercial array containing 19 different human cancer and normal tissues. The NOR1 expression levels were consistently high in nontumorous tissues but were underexpressed in the majority of paired tumor samples (Fig. 1). Notably, NOR1 expression was significantly
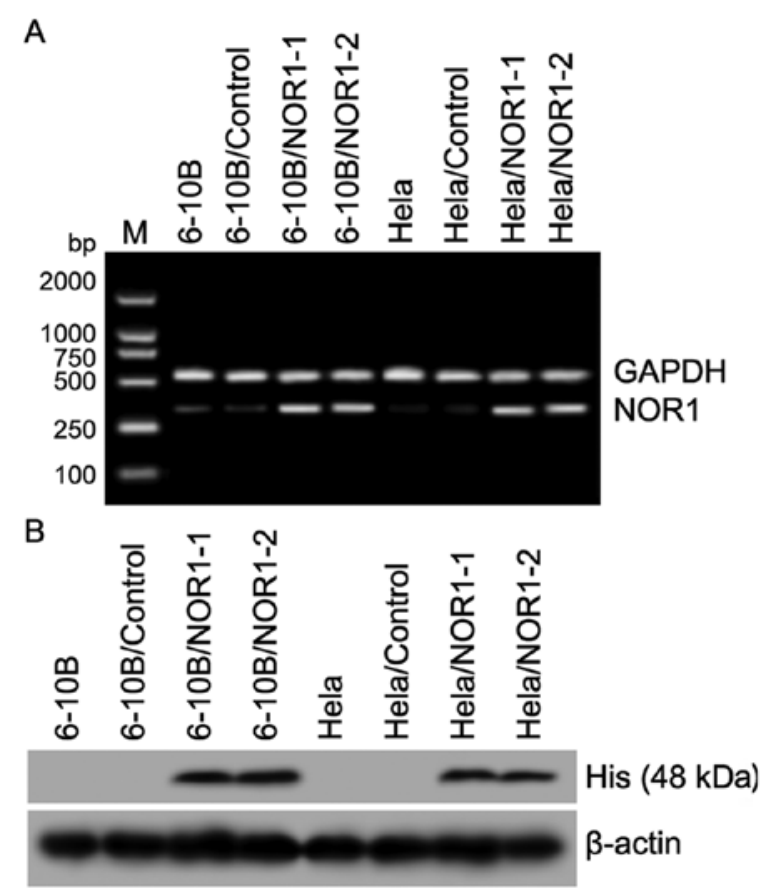

Figure 2. NOR1 mRNA and protein overexpressed in human NPC 6-10B/ NOR1 and CCA HeLa/NOR1 cells. The pCDNA3.1-myc-his-NOR1 or control vectors were stably transfected into 6-10B and HeLa cells. (A) NOR1 mRNA expression was detected by RT-PCR. GAPDH served as a control. (B) The expression of NOR1 protein was detected by western blotting with an anti-His antibody. $\beta$-actin served as loading control. All experiments were repeated three times with similar results.

decreased in 9 types of cancer tissues: ovary, lung, kidney, vulva, prostate, uterus, cervix, thyroid gland and testis. The other cancers represented on the array (i.e., breast, colon, stomach, bladder, rectum, skin, small intestine, pancreas, trachea and liver) did not show consistent upregulation or downregulation of NOR1 expression.

To systematically investigate the epidemiology of NOR1 expression in normal and neoplastic tissues, we used the TMAs to analyze the expression of NOR1 in 608 samples from five different malignant and normal tissue types (nasopharynx, lung, liver, gastric, colon and rectum) containing 348 cancer tissues, 172 adjacent normal tissues and 88 inflammatory tissues. ISH revealed a significantly lower rate of NOR1 mRNA expression in all five types of cancer tissues compared with the benign lesions $(\mathrm{P}<0.05)$ (Table I). However, there was no significant correlation between NOR1 mRNA expression and the clinical cancer stage. Therefore, these data clearly indicate NOR1 mRNA underexpression in NPC, lung, liver, gastric, colon and rectum cancers and suggest that its low expression may contribute to the pathogenesis of these cancers.

Stable expression of NORI in NPC 6-1OB and CCA HeLa cells after transfection with the pCDNA3.1-myc-his-NORI plasmid. Studies in our lab have shown that NOR1 expression is decreased in NPC and CCA tissues. In this study, we constructed the recombinant eukaryotic expression vector pCDNA3.1-mychis-NOR1 and transfected it into NPC 6-10B and CCA HeLa cells. NOR1 mRNA and protein were stably overexpressed in pCDNA3.1-myc-his-NOR1 transformants (pool clones) as 
Table I. NOR1 mRNA expression in five malignant and normal tissues analyzed by in situ hybridization.

\begin{tabular}{lcc}
\hline & \multicolumn{2}{c}{ NOR1 mRNA expression } \\
\cline { 2 - 3 } Tissue & Positive (\%) & Negative (\%) \\
\hline Nasopharyngeal & & \\
Cancer (n=148) & $49(33.1)^{\mathrm{a}}$ & $99(66.9)$ \\
Benign lesion (n=164) & $103(62.8)$ & $61(37.2)$ \\
Colon & & \\
Cancer (n=61) & $29(47.5)^{\mathrm{a}}$ & $32(52.5)$ \\
Benign lesion (n=32) & $28(87.5)$ & $4(12.5)$ \\
Lung & & \\
Cancer (n=89) & $48(53.9)^{\mathrm{a}}$ & $41(46.1)$ \\
Benign lesion (n=35) & $30(85.7)$ & $5(14.3)$ \\
Liver & & \\
Cancer (n=20) & $10(50.0)$ & $10(50.0)$ \\
Benign lesion (n=18) & $16(88.9)$ & $2(11.1)$ \\
Stomach & & \\
Cancer (n=30) & $15(50.0)^{\mathrm{a}}$ & $15(50.0)$ \\
Benign lesion $(\mathrm{n}=11)$ & $10(90.9)$ & $1(9.1)$ \\
Total & & \\
Cancer (n=348) & $151(43.4)^{\mathrm{a}}$ & $197(56.6)$ \\
Benign lesion (n=260) & $187(71.9)$ & $73(28.1)$ \\
\hline
\end{tabular}

${ }^{\mathrm{a} P}<0.05$.

confirmed by RT-PCR and western blotting (Fig. 2), and this provided an applicable cell model for further NOR1 functional analysis.

NORI overexpression in NPC 6-10B and CCA HeLa cells inhibits proliferation. An MTT assay demonstrated that the exogenous NOR1 significantly inhibited the proliferative capability of 6-10B and HeLa cells in NOR1-transfected cells as compared with untransfected negative controls and empty vector transfected groups $(\mathrm{P}<0.05)$ (Fig. 3A). Anchorage-independent growth in soft agar semisolid medium was a strong indicator of a transformed phenotype and a more stringent test of the mitogenic cancer capacity. In this study, NOR1 overexpression significantly weakened the potential of NPC and CCA cells to form colonies (Fig. 3B). Compared with the 6-10B and 6-10B/ Control groups, NOR1 overexpression led to an approximate $65.5 \%$ reduction in the number of colonies formed in 6-10B/ NOR 1 cells. The colony size and number was also consistently reduced when HeLa cells overexpressed NOR1 as compared with the two control groups.

To elucidate the impact of upregulated NOR1 expression on the cell cycle, FCM analysis was performed. As shown in Fig. 3C, the cells arrested in S phase when NOR1 was overexpressed. In 6-10B, 6-10B/Control, HeLa, and HeLa/Control groups, the cells in S phase accounted for 19.5, 21.4, 21.9 and $23.4 \%$, respectively. When NOR1 was overexpressed in 6-10B and HeLa cells, the cells in S phase accounted for 33.9 and
A
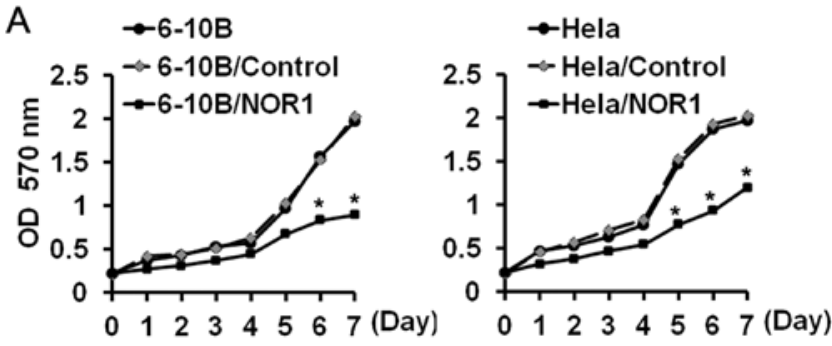

B
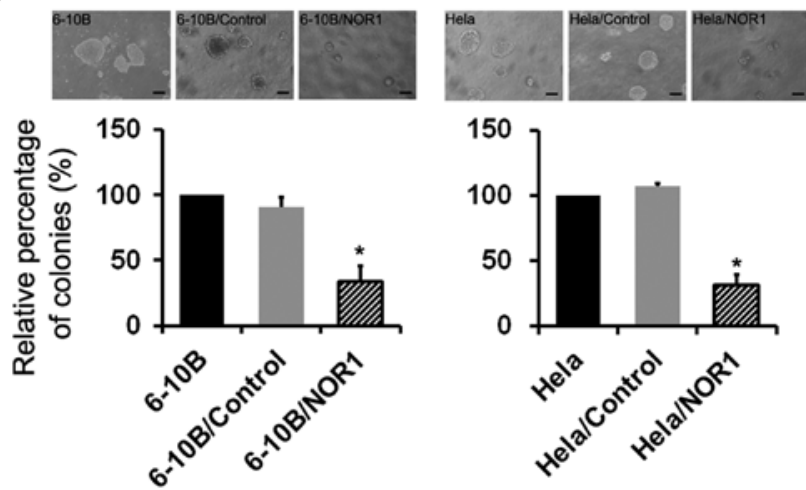

C
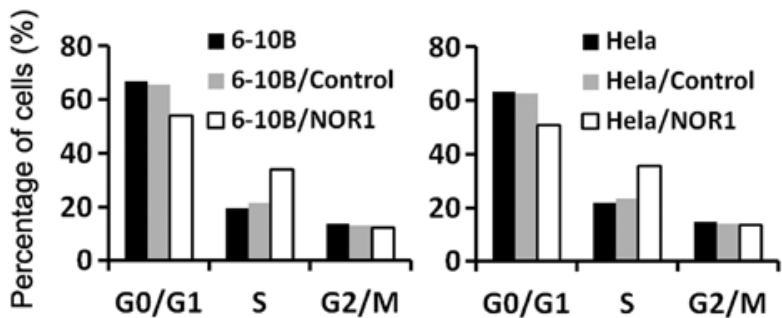

Figure 3. NOR1 overexpression in NPC 6-10B and CCA HeLa cells inhibits proliferation. (A) The MTT assay showed that NOR1 overexpression suppressed the growth of 6-10B and HeLa cells. (B) Colony formation assay indicated that NOR1 overexpression significantly reduced the colony formation rates of 6-10B and HeLa cells compared with those of the two control groups. Each bar represents the mean $\pm \mathrm{SE}$. ${ }^{*} \mathrm{P}<0.05$, compared with the two control groups. (C) NOR1 overexpression induced cell cycle arrest at $\mathrm{S}$ phase, as detected by FCM analysis. All experiments were repeated three times with similar results.

$35.7 \%$, respectively. This suggested that NOR1 overexpression arrested cells in $\mathrm{S}$ phase.

NORI overexpression in NPC 6-10B and CCA HeLa cells promotes apoptosis. We next explored the effect of NOR1 on apoptosis. Two methods were used to assess apoptosis: AO/EB staining and FCM analysis. AO/EB staining showed the characteristic changes of apoptotic morphology in 6-10B and HeLa cells overexpressing NOR1 (Fig. 4). Uniformly green live cells with normal morphology were observed in the control groups, whereas orange apoptotic cells with fragmented chromatin and apoptotic bodies were observed in 6-10B/NOR1 and HeLa/ NOR1 cells. These results suggested that NOR1 overexpression was able to induce a marked apoptotic morphology in NPC and CCA cells. FCM analysis results also revealed that $6-10 \mathrm{~B}$ and HeLa cells transfected with NOR1 contain a certain level of apoptotic cells $(1.97 \pm 0.22 \%$ and $2.39 \pm 0.45 \%$, respectively) which is more than that found in 6-10B, 6-10B/Control, HeLa and HeLa/Control cells $(0.27 \pm 0.11 \%, 0.21 \pm 0.09 \%, 0.16 \pm 0.13 \%$ and $0.17 \pm 0.11 \%$, respectively). There was no difference in the number of apoptotic cells between the two control groups. 


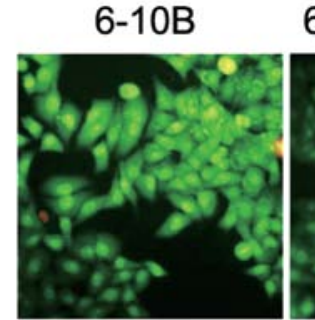

6-10B/Control
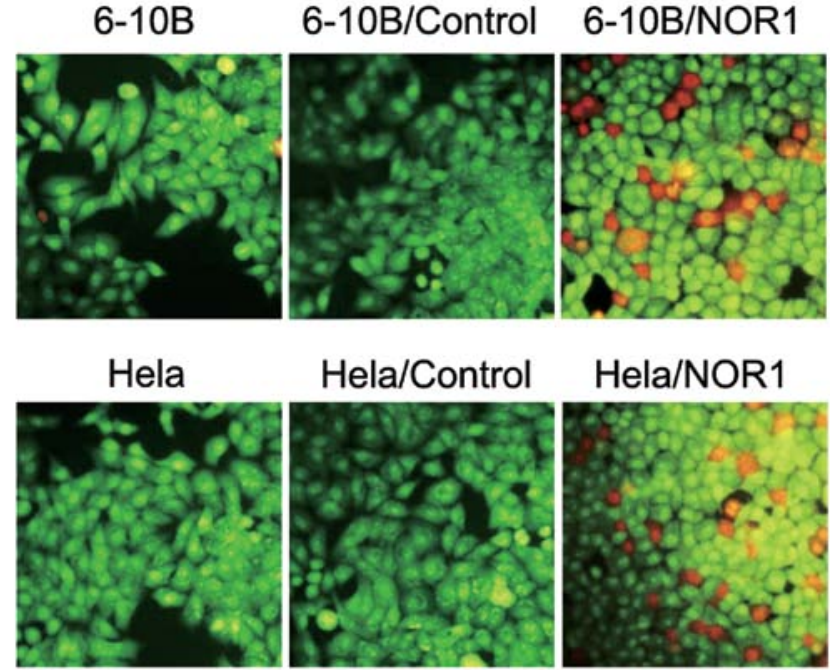

Figure 4. Apoptotic analysis. Apoptosis was detected by AO/EB staining. Green cells indicate live cells. Cells in which the nuclei are red or yellowred indicate apoptotic cells. All experiments were repeated three times with similar results.

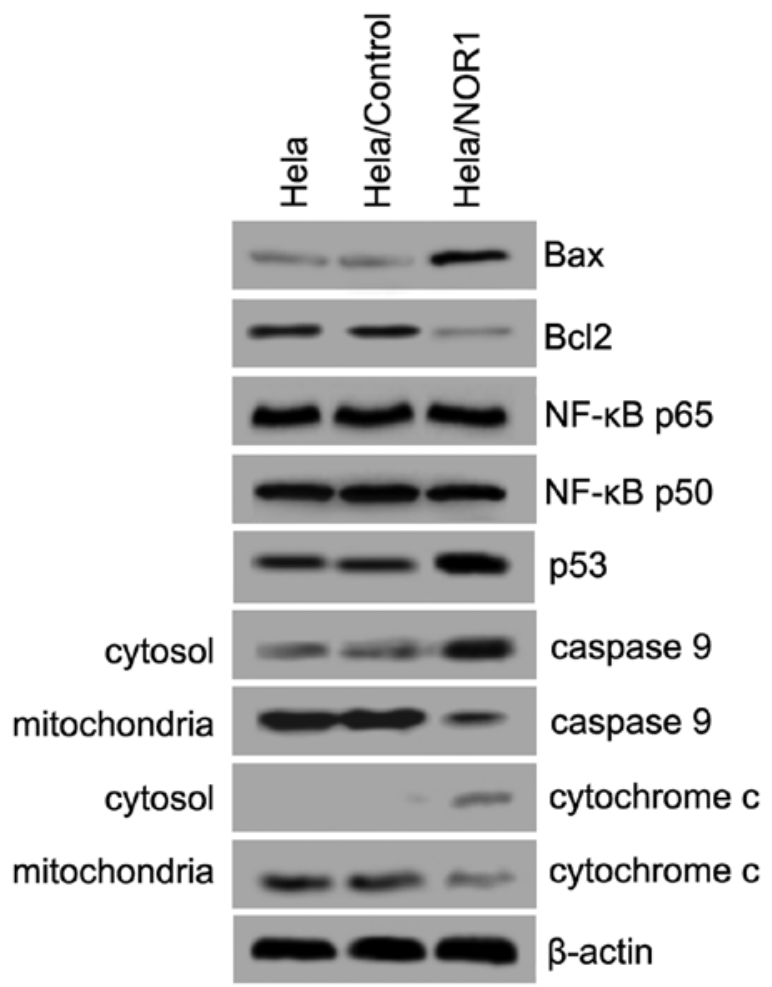

Figure 5. Apoptosis-related gene expression in NOR1-induced apoptosis. Western blot analysis of apoptosis-related genes in HeLa, HeLa/Control and $\mathrm{HeLa} / \mathrm{NOR} 1$ cells. $\beta$-actin served as a loading control. All experiments were repeated three times with similar results.

Apoptosis-related gene expression in NORI-induced apoptosis. We next investigated pathways that are potentially involved in NOR1-induced apoptosis. Western blot assays were performed using $\mathrm{HeLa} / \mathrm{NOR} 1$ cell lysates to examine the expression levels of Bax, Bcl-2, p53, and NF- $\mathrm{kB}$ and the release of cytochrome $c$ and caspase 9 from the mitochondria to cytosol. As shown in Fig. 5, NOR1 upregulation significantly
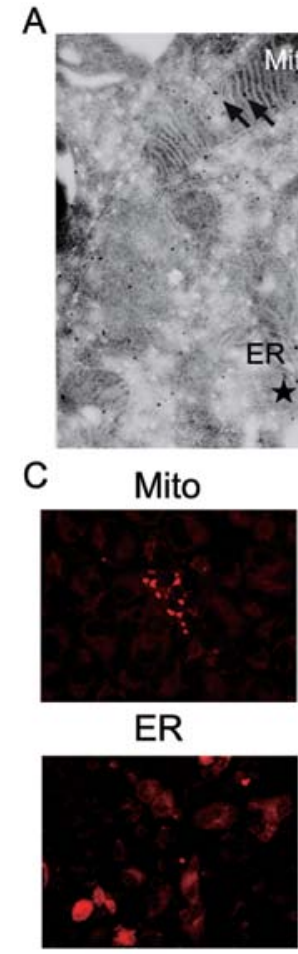

B
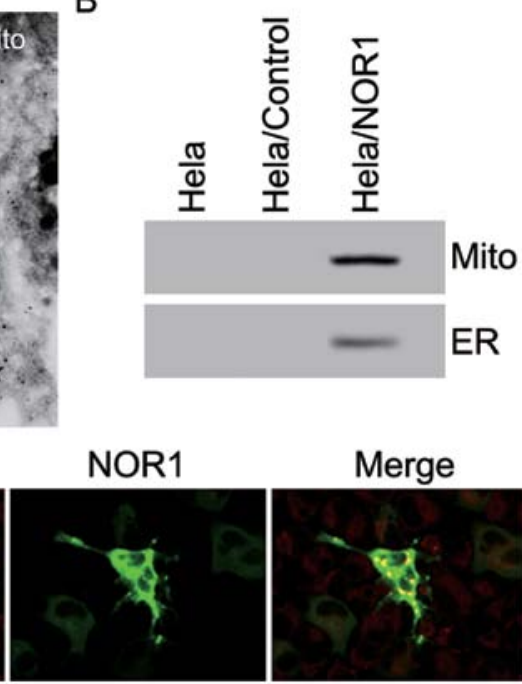

NOR1

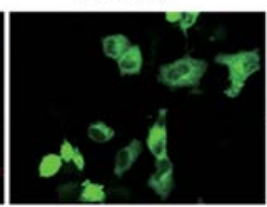

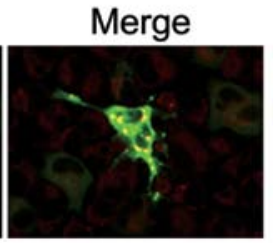

Merge

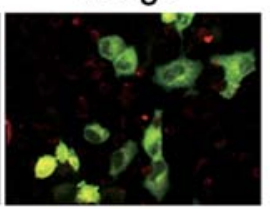

Figure 6. Intracellular localization of NOR1 in HeLa cells. (A) Subcellular localization of NOR1 by immune electron microscopic analysis with an anti-His antibody. NOR1 is localized in both the mitochondria (arrows) and ER (star). (B) Western blot analysis of the location of NOR1 in the mitochondria and ER with an anti-His antibody. (C) Upper panel: colocalization of mitochondria (red) and NOR1 (green). Lower panel: colocalization of ER (red) and NOR1 (green). Mito, mitochondria. ER, endoplasmic reticulum. All experiments were repeated three times with similar results.

led to an increased expression of Bax and p53 and a decreased expression of Bcl-2. However, NOR1 overexpression did not

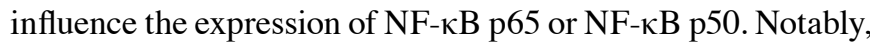
overexpressed NOR1 caused a decrease in expression of mitochondrial cytochrome $\mathrm{c}$ and caspase 9 , causing a concomitant increase in expression of cytosolic cytochrome $\mathrm{c}$ and caspase 9. Collectively, these results imply that NOR1-induced apoptosis in HeLa cells is mitochondria dependent.

Intracellular localization of NOR1 in HeLa cells. To further investigate the intracellular localization of NOR1 and its relationship with mitochondria, immune electron microscopy analysis was used. Immune electron microscopic analysis using an anti-His antibody confirmed that NOR1 is a cytoplasmic protein, which partially localizes to the mitochondria and ER (Fig. 6A). Next, the expression of NOR1 in the mitochondria and ER was assessed by western blotting (Fig. 6B). NOR1 protein was present in both the mitochondria and ER. pGFP-N1-NOR1 vectors were transiently transfected into HeLa cells, and after 24 hours, fluorescence microscopy was used to examine the subcellular localization of the NOR1 protein and mitochondria or ER in HeLa cells. Staining of HeLa/GFP-NOR1 cells with Mitotracker and ER-Tracker Red showed that NOR1 was localized to the cytoplasm and partly colocalized with the mitochondria and ER (Fig. 6C). Taken together, these results suggest that NOR1 is a cytoplasmic protein, which partly localizes to the mitochondria and ER. 


\section{Discussion}

The human NOR1 gene maps to human chromosome 1p34.2, which is a locus that is most frequently found to be lost in primary NPC biopsies (11-14). Previous studies have also revealed that the deletions of chromosomal arm $1 \mathrm{p}$ is frequent in oligodendroglial tumors, and this has been associated with a sensitivity to radio- and chemotherapy and favorable prognosis $(15,16)$. A high frequency of the loss of heterozygosity of $1 \mathrm{p} 34.2$ was associated with a higher grade of breast cancer in older women (17). Promoter hypermethylation-mediated silencing of tumor suppressor genes (TSGs) is a hallmark of oncogenesis. The NOR1 promoter region was found to be frequently methylated in NPC (3) and leukemia (4), and this may also lead to lower NOR1 expression in cancer cells. To understand whether NOR1 expression contributes to various types of cancers, NOR1 mRNA expression levels were quantified using the Cancer Profiling Array and TMAs. The results indicated underexpression of NOR1 mRNA in NPC, lung, liver, gastric, colon and rectum cancers and suggest that its low expression may contribute to the pathogenesis of various cancers.

To better understand the antitumor roles of NOR1, we constructed the recombinant expression vector pCDNA3.1-mychis-NOR1 and transfected it into NPC 6-10B and CCA HeLa cells, which appear to have low levels of NOR1 expression. Our study shows that NOR1 overexpression in NPC and CCA cells effectively inhibits cell proliferation and colony formation, arrests cells in $\mathrm{S}$ phase, and induces apoptosis in vitro, indicating a key role of NOR1 in the regulation of cell proliferation and apoptosis, which is consistent with its function as a tumor suppressor.

Moreover, our study is the first to prove the apoptosisrelative molecules involved in NOR1-induced apoptosis. Bcl-2 family members also play a critical role in the regulation of apoptosis. The Bcl-2 family, which is composed of both proapoptotic molecules (Bax, Bcl-Xs, Bak, Bid, Bad, Bim, and Bik) and anti-apoptotic molecules (Bcl-2, Bcl-XL, Bcl-W, Mcl-1, and A1), controls the release of mitochondrial cytochrome $\mathrm{c}$ by modulating the permeability of the outer mitochondrial membrane $(18,19)$. Herein, we showed that NOR1 may impact apoptosis through the activation or repression of downstream target genes. NOR1 overexpression attenuated the upregulation of Bax and decreased Bcl-2 expression, which resulted in mitochondrial dysfunction. Western blot analysis of cytochrome $\mathrm{c}$ and caspase 9 showed that NOR 1 overexpression induced cytochrome $\mathrm{c}$ and caspase 9 release from the mitochondria to the cytosol. These results suggest that NOR1induced apoptosis is mitochondria dependent. Furthermore, immune electron microscopy, western blotting and fluorescence staining analysis confirmed that NOR1 is a cytoplasmic protein that is partly localized to the mitochondria and ER. The role of the mitochondria-dependent apoptotic pathway in NOR1-induced apoptosis in cancer cells needs to be studied further.

Besides the Bcl-2 family members and the mitochondriadependent apoptotic pathway, NOR1 can also upregulate p53 protein expression. Previous studies have suggested that p53 is a central mediator for organizing cell responses to various stress and anticancer molecules with apoptosis, G1-arrest, and DNA repair (20). Nuclear factor $-\kappa B(N F-\kappa B)$ is also known for its anti-apoptotic function of transcriptional regulation of various anti-apoptotic genes involved in survival signaling $(21,22)$, and the relationship between $\mathrm{p} 53$ and $\mathrm{NF}-\kappa \mathrm{B}$ has been reported in recent years (23). p53 and the NF- $\mathrm{B}$-linked pathway were reported to be involved in various antitumor molecules that induced apoptosis (24-26). However, in this study, NOR1 overexpression did not influence the expression of NF- $\kappa \mathrm{B}$ p 65 and $\mathrm{NF}-\kappa \mathrm{B}$ p50. These data might be helpful to understand the molecular mechanism underlying NOR1 function in cancer cells, while the relationship between p53 in NOR1-induced apoptosis still needs to be further studied.

In conclusion, our findings demonstrate that NOR1 is underexpressed in NPC, lung, liver, gastric, colon and rectum cancers. Our results from the functional study of NOR1 at the cellular level further confirms that NOR1 functions as a tumor suppressor by inhibiting proliferation, preventing colony formation and promoting apoptosis. NOR1 is a cytoplasmic protein that is partially located in the mitochondria and endoplasmic reticulum, and the mitochondria-dependent apoptotic pathway is involved in NOR1-induced apoptosis. However, the precise mechanism behind the antitumor effects of NOR1 needs to be investigated further.

\section{References}

1. Nie X, Zhang B, Li X, et al: Cloning, expression, and mutation analysis of NOR1, a novel human gene down-regulated in HNE1 nasopharyngeal carcinoma cell line. J Cancer Res Clin Oncol 129: 410-414, 2003.

2. Xiang B, Yi M, Wang L, et al: Preparation of polyclonal antibody specific for NOR1 and detection of its expression pattern in human tissues and nasopharyngeal carcinoma. Acta Biochim Biophys Sin 41: 754-762, 2009.

3. Li W, Li X, Wang W, et al: NOR1 is an HSF1- and NRF1regulated putative tumor suppressor inactivated by promoter hypermethylation in nasopharyngeal carcinoma. Carcinogenesis 32: 1305-1314, 2011.

4. Kroeger H, Jelinek J, Estecio MR, et al: Aberrant CpG island methylation in acute myeloid leukemia is accentuated at relapse. Blood 112: 1366-1373, 2008.

5. Calmels S, Ohshima H, Rosenkranz H, McCoy E and Bartsch H: Biochemical studies on the catalysis of nitrosation by bacteria. Carcinogenesis 8: 1085-1088, 1987.

6. Reznik G, Mohr U and Kruger FW: Carcinogenic effects of Di-npropylnitrosamine, beta-hydroxypropyl-n-propylnitrosamine, and methyl-n-propylnitrosamine on Sprague-Dawlay rats. J Natl Cancer Inst 54: 937-943, 1975.

7. Lijinsky W and Taylor HW: Carcinogenicity of methylated nitrosopiperidines. Int J Cancer 16: 318-322, 1975.

8. Chang ET and Adami HO: The enigmatic epidemiology of nasopharyngeal carcinoma. Cancer Epidemiol Biomarkers Prev 15: 1765-1777, 2006.

9. Lo KW, To KF and Huang DP: Focus on nasopharyngeal carcinoma. Cancer Cell 5: 423-428, 2004.

10. Lin YP, Nicholas K, Ball FR, McLaughlin B and Bishai FR: Detection of Norwalk-like virus and specific antibody by immuneelectron microscopy with colloidal gold immune complexes. J Virol Methods 35: 237-253, 1991.

11. Yan J, Fang Y and Liang Q: Frequent chromosomal gain of $4 \mathrm{q}$ and loss of $1 \mathrm{p}$ in primary nasopharyngeal carcinoma. Zhonghua Zhong Liu Za Zhi 23: 208-210, 2001 (In Chinese).

12. Fang Y, Guan X, Guo Y, et al: Analysis of genetic alterations in primary nasopharyngeal carcinoma by comparative genomic hybridization. Genes Chromosomes Cancer 30: 254-260, 2001.

13. Shao JY, Wang HY, Huang XM, et al: Genome-wide allelotype analysis of sporadic primary nasopharyngeal carcinoma from southern China. Int J Oncol 17: 1267-1275, 2000.

14. Lo KW, Teo PM, Hui AB, et al: High resolution allelotype of microdissected primary nasopharyngeal carcinoma. Cancer Res 60: 3348-3353, 2000. 
15. Gadji M, Fortin D, Tsanaclis AM and Drouin R: Is the $1 \mathrm{p} / 19 \mathrm{q}$ deletion a diagnostic marker of oligodendrogliomas? Cancer Genet Cytogenet 194: 12-22, 2009.

16. Tews B, Roerig P, Hartmann C, et al: Hypermethylation and transcriptional downregulation of the CITED4 gene at $1 \mathrm{p} 34.2$ in oligodendroglial tumours with allelic losses on $1 \mathrm{p}$ and $19 \mathrm{q}$. Oncogene 26: 5010-5016, 2007.

17. Chunder N, Mandal S, Basu D, Roy A, Roychoudhury S and Panda CK: Deletion mapping of chromosome 1 in early onset and late onset breast tumors - a comparative study in eastern India. Pathol Res Pract 199: 313-321, 2003.

18. Jung JY and Kim WJ: Involvement of mitochondrial- and Fas-mediated dual mechanism in $\mathrm{CoCl}_{2}$-induced apoptosis of rat PC12 cells. Neurosci Lett 371: 85-90, 2004.

19. Gross A, McDonnell JM and Korsmeyer SJ: BCL-2 family members and the mitochondria in apoptosis. Genes Dev 13: $1899-1911,1999$.

20. Kruse JP and Gu W: Modes of p53 regulation. Cell 137: 609-622, 2009.

21. Wang CY, Mayo MW, Korneluk RG, Goeddel DV and Baldwin AS Jr: NF-kappaB antiapoptosis: induction of TRAF1 and TRAF2 and c-IAP1 and c-IAP2 to suppress caspase-8 activation. Science 281: 1680-1683, 1998.
22. Kucharczak J, Simmons MJ, Fan Y and Gelinas C: To be, or not to be: NF-kappaB is the answer - role of Rel/NF-kappaB in the regulation of apoptosis. Oncogene 22: 8961-8982, 2003.

23. Stoffel A and Levine AJ: Actvation of NF-kappaB by the API2/ MALT1 fusions inhibits p53 dependant but not FAS induced apoptosis: a directional link between NF-kappaB and p53. Cell Cycle 3: 1017-1020, 2004.

24. Yin Y, Chen W, Tang C, et al: NF-kappaB, JNK and $\mathrm{p} 53$ pathways are involved in tubeimoside-1-induced apoptosis in HepG2 cells with oxidative stress and $\mathrm{G}(2) / \mathrm{M}$ cell cycle arrest. Food Chem Toxicol 49: 3046-3054, 2011

25. Maldonado ME, Bousserouel S, Gosse F, Lobstein A and Raul F: Implication of NF-kappaB and p53 in the expression of TRAILdeath receptors and apoptosis by apple procyanidins in human metastatic SW620 cells. Biomedica 30: 577-586, 2010.

26. Pratheeshkumar P, Sheeja K and Kuttan G: Andrographolide induces apoptosis in B16F-10 melanoma cells by inhibiting NF-kappaB-mediated bcl-2 activation and modulating p53-induced caspase-3 gene expression. Immunopharmacol Immunotoxicol 34: $143-151,2012$. 